\title{
The Effect of Transit Signal Priority on Bus Rapid Transit Headway Adherence
}

\author{
Gregory S. Macfarlane ${ }^{1}$ (D) ${ }^{\text {a }}$, Michael H. Sheffield ${ }^{2}$, Logan S. Bennett ${ }^{1}$, Grant G. Schultz ${ }^{1}$ (D) \\ ${ }^{1}$ Civil and Environmental Engineering, Brigham Young University, ${ }^{2}$ Wall Consultant Group \\ Keywords: public transit, transit signal priority, traffic operations \\ https://doi.org/10.32866/001c.24499
}

\section{Findings}

\begin{abstract}
We report the results of an experiment to evaluate the impact of transit signal priority (TSP) on headway adherence for a bus rapid transit (BRT) system in Provo / Orem, Utah. The bus requests TSP based on its unpublished schedule, but users perceive only a headway. Quantile regression models estimated on raw timepoint data from the BRT system reveal that TSP significantly improves headway adherence, after controlling for peak times, direction, and cumulative trip dwell time. We also find that requiring the bus to be 2 minutes late before requesting TSP improves headway adherence more than allowing all buses to request TSP.
\end{abstract}

\section{Questions}

Transit signal priority (TSP) allows traffic signals to flexibly accommodate transit vehicles. This may involve extending a green phase until the vehicle passes, triggering an early green if there is a vehicle waiting at the light, or even running specific transit-only phases. TSP helps transit vehicles maintain on-time performance (Sheffield et al. 2021; Liu et al. 2018), but often TSP will only engage at a signal if the vehicle is running behind its schedule, thus minimizing automobile delay when the bus is otherwise on schedule (Ni et al. 2020).

In 2018, the Utah Transit Authority (UTA) launched the Utah Valley Express (UVX) Bus Rapid Transit (BRT) system in Provo and Orem, Utah. The system - mapped in Figure 1 - connects two commuter rail stations, two major universities (Brigham Young and Utah Valley), and commercial districts in Orem and Provo. UVX has TSP on 44 of the 47 traffic signals along its route. A transit vehicle requests TSP when the vehicle is behind its schedule; the signal controller may then grant the TSP based on a set of rules. For example, the signal will not grant TSP to transit vehicles traveling in the same direction in consecutive signal cycles. Specifically, at least two "clean" cycles must run before the signal controller will grant TSP again.

Though the TSP system is based on a schedule, UTA does not publish a schedule for UVX. Instead it is expected that a bus will arrive on a specific headway: 6 minutes in the peak period and 10 minutes in the off-peak. UVX vehicles depart their initial time point on this schedule, but then proceed to 


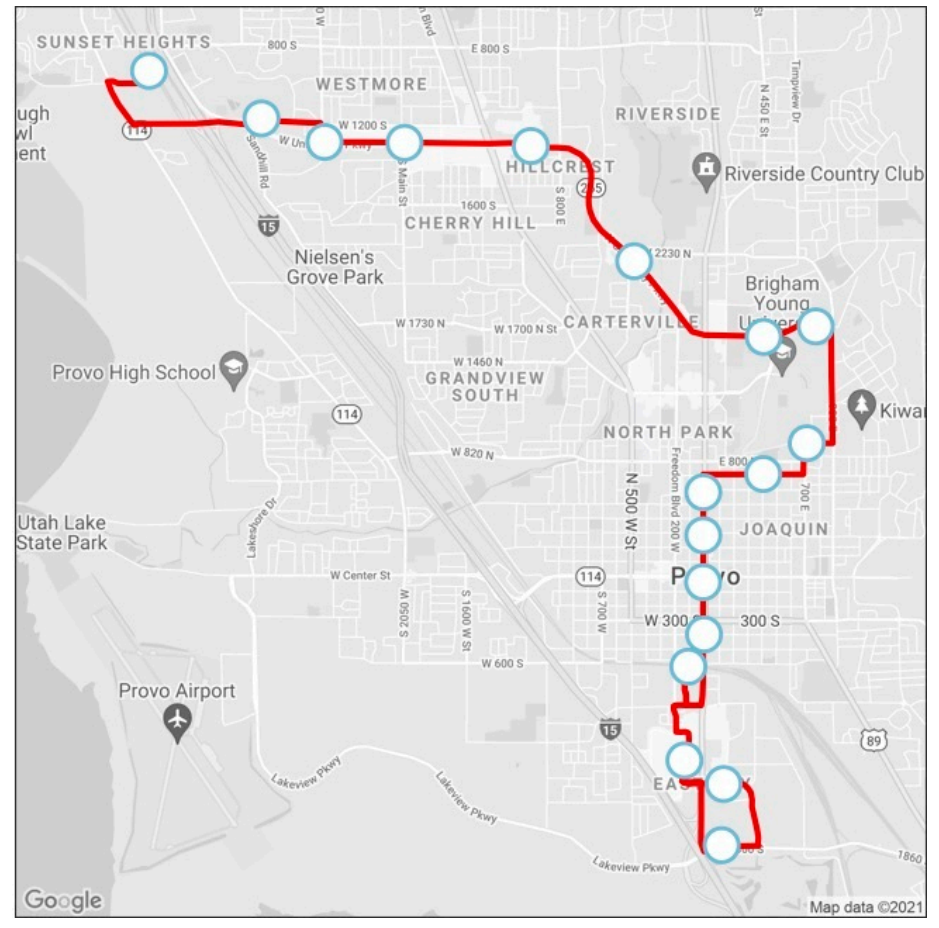

Figure 1. UVX system stations and alignment

the end of the route without consideration as to whether the vehicle is running ahead or behind its "schedule" as understood by the TSP system. It is unclear whether the actualized headway is affected by a schedule-based TSP system, potentially creating frustration for riders or inefficient operations. The research questions are therefore:

- Does schedule-based TSP improve headway adherence for rapid transit systems?

- What is the effect of other controlling factors - specifically time period, direction of travel, and cumulative stop dwell time - on headway adherence?

\section{Methods}

UTA provided time point data for all trips on the UVX system during the summer of 2019. During this period, the TSP requesting threshold was set at three different levels:

- June 10 through July 12 and after August 12: 2 minute threshold

- July 15 through July 26: no TSP

- July 30 through August 9: TSP always requested

We calculated the headway between successive UVX trips at each stop, as well as the cumulative dwell time of all stations along the route to that point. To control for omitted variables, we limited our analysis to the periods between 
$7 \mathrm{AM}$ and $8 \mathrm{PM}$ when the system runs at a 6-minute headway. Time points within these periods are considered "AM Peak" if occurring between 7 and 9 AM, or "PM Peak" between 4 and 6 PM. We also discard timepoints in south Provo where UVX runs on a one-way circulating loop.

Standard statistical tests - such as the student's $t$-test or ordinary least squares regression models - are designed to ascertain the significance of a statistic at the mean of the distribution. In this application, we are less concerned with the mean deviation in headway, and are instead interested in whether TSP is able to reduce the lateness of buses that already have substantial deviation from their programmed headway. Further, a bus that is delayed from its intended headway may shorten the subsequent headway due to "bunching." Consequently, we employ conditional quantile regression (Koenker and Hallock 2001) to estimate the effect of TSP requesting threshold on headway deviation at multiple percentiles of the distribution. This is done with the quantreg package for R (Koenker 2020; R Core Team 2021).

Raw data and complete analysis code are available in a public GitHub repository.

\section{Findings}

Figure 2 shows the empirical cumulative density function for the headway deviation data, grouped by TSP threshold. Note that a "perfect" headway distribution, where all vehicles maintain the exactly intended headway, would be a step function with a vertical transition at 6 minutes. Visually, the difference between the various threshold settings is not dramatic. The 2-minute threshold appears to have slightly more vehicles arrive behind the scheduled headway (6 minutes), and slightly more arrive before it, than the other two threshold groups. The median of the distribution for all three thresholds is remarkably similar and is just a few seconds behind the target headway. This observation strengthens our determination to examine the sides of the distribution - rather than its center - with a quantile regression model.

Models estimating the headway effects of TSP requesting threshold and other factors at an array of distribution quantiles are given in Table 1. In these models, the "(Intercept)" represents the expected headway at that percentile before considering additional information. For example, the 10th-percentile headway is 4.649 minutes ( 4 minutes and 39 seconds), all else equal. The other coefficients in the models modify this average headway. Almost all coefficients are significant, and many serve to widen the headway distribution. Buses traveling in the PM peak, for example, have a significantly higher 90thpercentile headway and a lower 10th-percentile headway than buses traveling outside of the AM or PM peaks. Buses traveling in the southbound direction also have a wider headway distribution than those in the northbound direction (implied to be 0 ), and each additional minute of cumulative dwell time widens the headway distribution. 


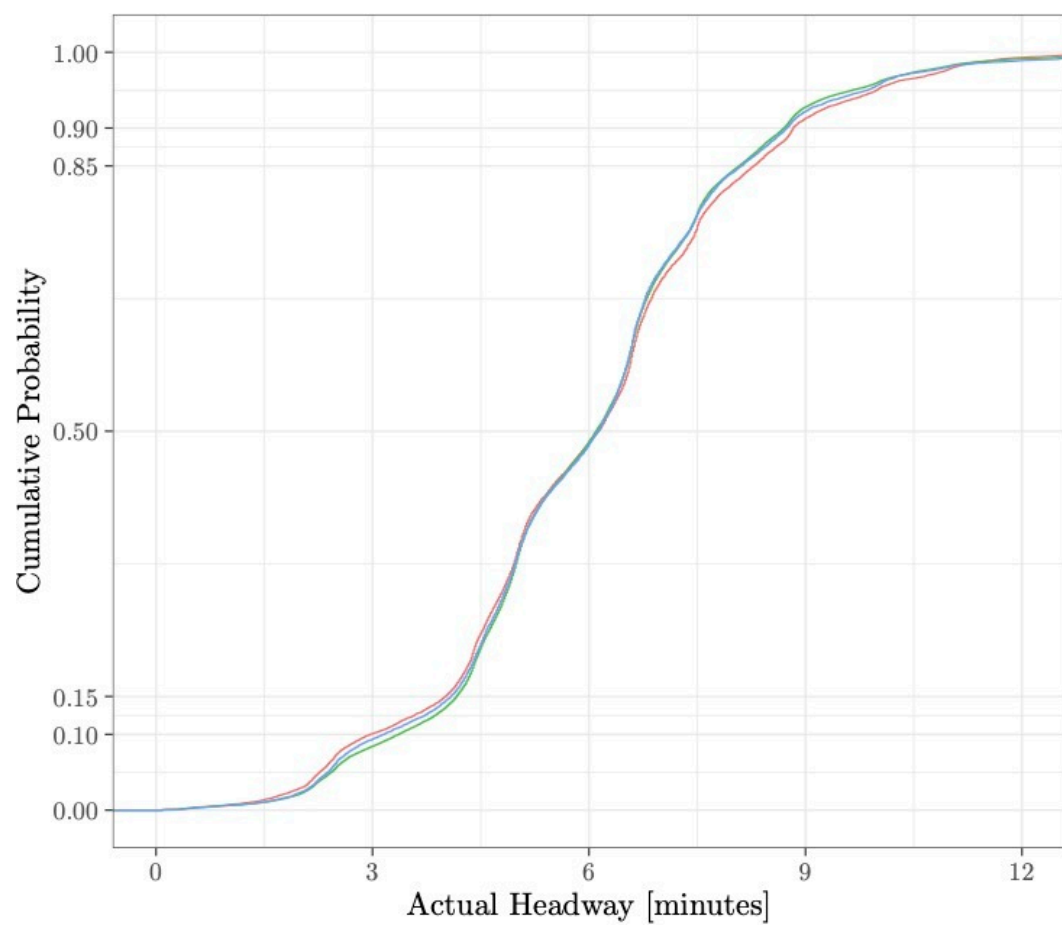

TSP Threshold

- No TSP

- $2 \mathrm{~min}$

- Always

Figure 2. Cumulative probability distribution of headway deviation by threshold

Table 1. Quantile Regression Estimates

\begin{tabular}{|c|c|c|c|c|c|}
\hline & 10th & 15th & 50th & 85th & 90th \\
\hline (Intercept) & $\begin{array}{c}4.649 \\
(108.947)^{* * *}\end{array}$ & $\begin{array}{c}4.922 \\
(131.183)^{* * *}\end{array}$ & $\begin{array}{c}6.338 \\
(174.147)^{* * *}\end{array}$ & $\begin{array}{c}7.410 \\
(187.349)^{* * *}\end{array}$ & $\begin{array}{c}7.598 \\
(209.077)^{* * *}\end{array}$ \\
\hline TSP: 2 minutes & $\begin{array}{c}0.190 \\
(5.234)^{* * *}\end{array}$ & $\begin{array}{c}0.168 \\
(4.880)^{* * *}\end{array}$ & $\begin{array}{c}-0.013 \\
(-0.400)\end{array}$ & $\begin{array}{c}-0.186 \\
(-5.346)^{* * *}\end{array}$ & $\begin{array}{c}-0.178 \\
(-5.399)^{* * *}\end{array}$ \\
\hline TSP: Always & $\begin{array}{c}0.093 \\
(2.033)^{* *}\end{array}$ & $\begin{array}{c}0.101 \\
(2.479)^{* *}\end{array}$ & $\begin{array}{c}0.001 \\
(0.018)\end{array}$ & $\begin{array}{c}-0.218 \\
(-4.650)^{* * *}\end{array}$ & $\begin{array}{c}-0.132 \\
(-2.835)^{* * *}\end{array}$ \\
\hline Southbound & $\begin{array}{c}-0.921 \\
(-21.746)^{* * *}\end{array}$ & $\begin{array}{c}-0.604 \\
(-17.391)^{* * *}\end{array}$ & $\begin{array}{c}-0.161 \\
(-4.591)^{* * *}\end{array}$ & $\begin{array}{c}0.752 \\
(21.580)^{* * *}\end{array}$ & $\begin{array}{c}0.861 \\
(26.837)^{* * *}\end{array}$ \\
\hline AM Peak & $\begin{array}{c}-0.160 \\
(-5.148)^{* * *}\end{array}$ & $\begin{array}{c}-0.114 \\
(-3.578)^{* * *}\end{array}$ & $\begin{array}{c}0.075 \\
(2.973)^{* * *}\end{array}$ & $\begin{array}{c}-0.068 \\
(-1.211)\end{array}$ & $\begin{array}{c}0.112 \\
(3.453)^{* * *}\end{array}$ \\
\hline PM Peak & $\begin{array}{c}-0.444 \\
(-8.855)^{* * *}\end{array}$ & $\begin{array}{c}-0.204 \\
(-4.895)^{* * *}\end{array}$ & $\begin{array}{c}-0.401 \\
(-6.218)^{* * *}\end{array}$ & $\begin{array}{c}0.380 \\
(9.390)^{* * *}\end{array}$ & $\begin{array}{c}0.461 \\
(12.282)^{* * *}\end{array}$ \\
\hline Cumulative Dwell [minutes] & $\begin{array}{c}-0.205 \\
(-44.282)^{* * *}\end{array}$ & $\begin{array}{c}0.461 \\
(12.282)^{* * *}\end{array}$ & $\begin{array}{c}-0.041 \\
(-8.505)^{* * *}\end{array}$ & $\begin{array}{c}0.105 \\
(15.956)^{* * *}\end{array}$ & $\begin{array}{c}0.161 \\
(31.324)^{* * *}\end{array}$ \\
\hline Southbound x AM Peak & $\begin{array}{c}0.004 \\
(0.063)\end{array}$ & $\begin{array}{c}-0.067 \\
(-1.144)\end{array}$ & $\begin{array}{c}0.141 \\
(2.876)^{* * *}\end{array}$ & $\begin{array}{c}0.415 \\
(6.201)^{* * *}\end{array}$ & $\begin{array}{c}0.257 \\
(4.611)^{* * *}\end{array}$ \\
\hline Southbound $x$ PM Peak & $\begin{array}{c}0.285 \\
(4.076)^{* * *}\end{array}$ & $\begin{array}{c}-0.286 \\
(-5.420)^{* * *}\end{array}$ & $\begin{array}{c}-0.003 \\
(-0.032)\end{array}$ & $\begin{array}{c}-0.171 \\
(-2.371)^{* *}\end{array}$ & $\begin{array}{c}-0.157 \\
(-2.470)^{* *}\end{array}$ \\
\hline AIC & $321,907.1$ & $314,210.7$ & $301,346.1$ & $325,312.4$ & $336,009.8$ \\
\hline Log Likelihood & $-160,944.5$ & $-157,096.3$ & $-150,664$ & $-162,647.2$ & $-167,995.9$ \\
\hline
\end{tabular}

t-statistics in parentheses, ${ }^{*} \mathrm{p}<0.1,{ }^{* *} \mathrm{p}<0.05,{ }^{* * *} \mathrm{p}<0.01$

Coefficients represent change to expected headway in minutes

In contrast, the estimates reveal that TSP significantly narrows the expected headway distribution, with fewer long headways and fewer short headways. And while most of the other explanatory variables have an effect on the median headway, TSP has no significant effect after these other variables have been 
controlled for. A potentially curious finding is that implementing a 2-minute TSP request threshold improves headway adherence more than allowing every transit vehicle to request TSP. This finding echoes the schedule-based TSP analysis of UVX by Schultz et al. (2020). A finding by Sheffield et al. (2021) that a 0-minute threshold is best on arterial bus systems may only apply to routes with longer headways.

The schedule of threshold changes was not randomized in any way, and it is possible that the results of this study are tied up in unaccounted seasonal variation, or other omitted explanatory variables. These limitations notwithstanding, we find that - all else equal - schedule-based TSP marginally improves the headway adherence of UVX.

\section{Acknowledgements}

We are grateful to the Utah Department of Transportation and UTA for supporting this research by providing data and configuring the experiment. Graphics and tables were produced with several open-source $R$ packages (Kahle and Wickham 2013; Arel-Bundock 2021; Ram and Wickham 2018). 


\section{REFERENCES}

Arel-Bundock, V. 2021. "Modelsummary: Summary Tables and Plots for Statistical Models and Data: Beautiful, Customizable, and Publication-Ready.” 2021.

https://vincentarelbundock.github.io/modelsummary/.

Kahle, D., and H. Wickham. 2013. "Ggmap: Spatial Visualization with Ggplot2.” The R Journal 5: 144-61. https://journal.r-project.org/archive/2013-1/kahle-wickham.pdf.

Koenker, R. 2020. “Quantreg: Quantile Regression.” R package version 5.55. 2020.

https://CRAN.R-project.org/package=quantreg.

Koenker, R., and Kevin F. Hallock. 2001. "Quantile Regression.” Journal of Economic Perspectives 15 (4): 143-56. https://doi.org/10.1257/jep.15.4.143.

Liu, X.C., M. Zlatkovic, R. J. Porter, K. Fayyaz, and S. Yu. 2018. "Improving Efficiency and Reliability of Bus Rapid Transit.” Technical Report MPC 18-349. Mountain-Plains Consortium.

$\mathrm{Ni}$, Ying-Chuan, Hsien-Hao Lo, Yu-Ting Hsu, and Hung-Jen Huang. 2020. "Exploring the Effects of Passive Transit Signal Priority Design on Bus Rapid Transit Operation: A MicrosimulationBased Optimization Approach.” Transportation Letters, August, 1-14. https://doi.org/10.1080/ $\underline{19427867.2020 .1805681 .}$.

R Core Team. 2021. "R: A Language and Environment for Statistical Computing.” R Foundation for Statistical Computing Vienna, Austria. 2021. https://www.R-project.org/.

Ram, K., and H. Wickham. 2018. "Wesanderson: A Wes Anderson Palette Generator." R package version 0.3.6. 2018. https://CRAN.R-project.org/package=wesanderson.

Schultz, G. G., M. H. Sheffield, D. Bassett, and D. L. Eggett. 2020. "Impacts of Changing the Transit Signal Priority Requesting Threshold on Bus Performance and General Traffic: A Sensitivity Analysis.” Technical Report UT-20.06. Utah Department of Transportation Research \& Innovation Division.

Sheffield, Michael H., Grant G. Schultz, David Bassett, and Dennis L. Eggett. 2021. "Sensitivity Analysis of the Transit Signal Priority Requesting Threshold and the Impact on Bus Performance and General Traffic.” Transportation Research Record, January. https://doi.org/10.1177/ $\underline{0361198120985853}$. 